\title{
Controlling turbulence in present and future stellarators
}

\author{
P. Xanthopoulos ${ }^{1 *}$, H. E. Mynick ${ }^{2}$, P. Helander ${ }^{1}$, Y. Turkin ${ }^{1}$, G. G. Plunk ${ }^{1}$, \\ F. Jenko ${ }^{3}$, T. Görler ${ }^{3}$, D. Told ${ }^{3}$, T. Bird ${ }^{1}$ and J. H. E. Proll ${ }^{1}$ \\ ${ }^{1}$ Max-Planck-Institut für Plasmaphysik, \\ Wendelsteinstr. 1, 17491 Greifswald, Germany \\ 2 Plasma Physics Laboratory, Princeton University, \\ Princeton, New Jersey 08543, USA \\ ${ }^{3}$ Max-Planck-Institut für Plasmaphysik, \\ Boltzmannstr. 2, 85748 Garching, Germany \\ *e-mail: pavlos.xanthopoulos@ipp.mpg.de
}

\begin{abstract}
Turbulence is widely expected to limit the confinement, and thus the overall performance, of modern neoclassically-optimized stellarators. We employ novel petaflop-scale gyrokinetic simulations to predict the distribution of turbulence fluctuations and the related transport scaling on entire stellarator magnetic surfaces, and reveal striking differences to tokamaks. Using a stochastic global-search optimization method, we derive the first turbulence-optimized stellarator configuration stemming from an existing quasi-omnigenous design.
\end{abstract}

Throughout the history of magnetic fusion, a recurrent theme has been the surprising sensitivity of plasma performance to the details of the magnetic field. For instance, it has long been known that the confinement of alpha particles can be spoiled by small ripples in the magnetic field. More recently, magnetic perturbations have been found to dramatically influence instabilities of the plasma edge [1]. In both stellarators and tokamaks, experiments show that the level of turbulence may be reduced by modifying the magnetic field. As notable examples, the confinement time in the TCV tokamak is doubled by reversing the triangularity of the poloidal cross section of the flux surfaces [2], and in the LHD stellarator the turbulent transport can be reduced significantly by adjusting the coil currents so as to shrink the circumference of the torus by pushing it radially inwards [3].

Stellarators typically possess $40-50$ degrees of freedom in the shaping of the magnetic field, an order of magnitude more than for tokamaks [4]. This enhanced flexibility can be used to optimize various plasma properties [5], and the latest demonstration of the power of such optimization is expected to be realized in the superconducting stellarator experiment Wendelstein 7-X (W7$\mathrm{X}$ ), in Greifswald, Germany [6]. A tantalizing possibility for fusion researchers is to try to exploit any leeway in the magnetic geometry to design configurations with better confinement. In W7-X, this has already been done for the collisional (so-called "neoclassical") transport, but no device built so far is optimized with respect to turbulence.

In order to understand how energy transport depends on the magnetic-field geometry, it is helpful to numerically simulate the turbulence in a large portion of the plasma. In tokamaks, thanks to axisymmetry, restricting the computational domain to a "flux tube", a slender volume encompassing a magnetic-field line [7], suffices to calculate the transport at a radial location. In a stellarator, however, different flux tubes on a magnetic surface are not geometrically equivalent, thus it appears necessary to simulate the entire magnetic surface. Much has been learned from the flux-tube approach which, except for inspiring efforts [8], has characterized stellarator turbulence simulations to date $[9-12]$. Nevertheless, all these simulations have a major inherent drawback: the transport cannot be reliably determined, as the turbulence strength generally varies between different flux tubes on the same stellarator magnetic surface. The new "flux-surface" version of the GENE code [1315] - a massively-parallel Eulerian solver of the nonlinear gyrokinetic system of equations $[16,17]$ - has the unique capability of simulating turbulence on an arbitrary toroidal magnetic surface (whilst still employing a local approximation in the radial direction), thus overcoming this problem.

In Fig. 1, the result of a flux-surface GENE simulation of turbulence in $\mathrm{W} 7-\mathrm{X}$ is presented. The plasma ions are treated gyrokinetically while the electrons are assumed to have a Boltzmann distribution, and the turbulence is driven by the ion temperature gradient (ITG). The strongest fluctuations are localized along a thin stripe on the outboard side of W7-X, leaving the rest of the surface relatively quiescent (we note in passing that a similar localization is predicted also for a quasi-axisymmetric stellarator configuration). This remarkable feature, which is not found in tokamaks, affects the scaling of the transport with the parameter $\rho^{*}=\rho_{i} / a$, where $\rho_{i}$ denotes the gyroradius of thermal ions and $a$ is the (averaged) minor radius of the device. When $\rho^{*} \rightarrow 0$ holds, all transport coefficients become inversely proportional to the square of the magnetic field, something known as "gyro-Bohm scaling". This limit is observed in flux-tube simulations for tokamaks as well as stellarators, but the flux-surface GENE simulations of W7-X turbulence in Fig. 2 indicate that the transport deviates from this scaling for experimentally relevant values of $\rho^{*}$. It is found that the uneven distribution of curvature on the stellarator surface causes the localization of turbulence by imposing a characteris- 


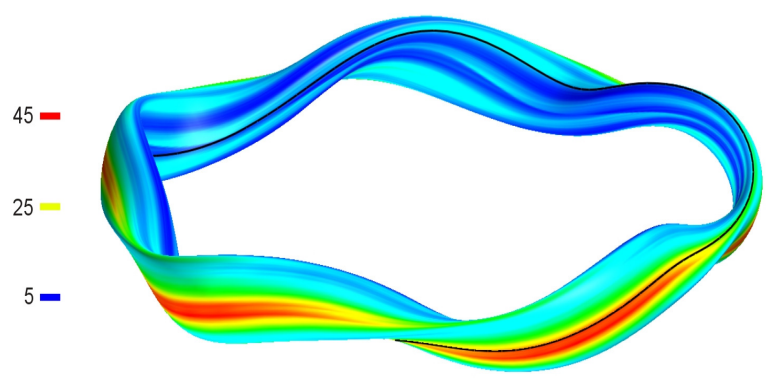

FIG. 1. Root-mean-squared electrostatic potential fluctuations caused by ion-temperature-gradient-driven turbulence in units of $T_{i} \rho^{*} / e$, where $T_{i}$ is the ion temperature, $e$ is the electron charge and $\rho^{*}=1 / 125$ is the normalized ion gyroradius. The strongest fluctuations are contained in the red stripe on the outboard side of the surface. The resolution for the $5 \mathrm{D}$ simulation box reads $\left(\hat{L}_{r} / N_{r}, \hat{L}_{y} / N_{y}, \hat{L}_{z} / N_{z}, \hat{L}_{v} / N_{v}, \hat{L}_{\mu} / N_{\mu}\right)=$ $(177 / 128,128 / 480,2 \pi / 128,3 / 64,9 / 8)$, where $r, y, z$ are the spatial (radial, binormal, parallel) dimensions, $v$ is the velocity parallel to the magnetic field and $\mu$ is the magnetic moment. $\hat{L}_{r}$ is the dimensionless length and $N_{r}$ the number of discretization points in the radial direction etc. The normalized ion temperature gradient is $-a / T_{i} d T_{i} / d r=2$.

tic length $L_{c}$, corresponding to the variation of curvature on the surface in the direction perpendicular to the magnetic field. For W7-X, this length is very close to the minor radius and thus $\rho^{*}$ is a pertinent scaling parameter. In a tokamak, on the other hand, $L_{c}$ is roughly half

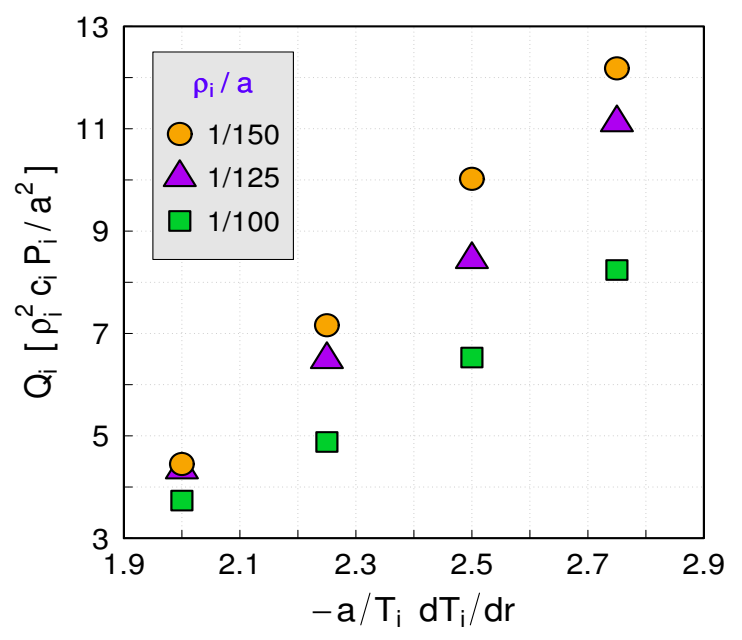

FIG. 2. (Color online) Ion-heat-flux scaling for different $\rho^{*}$ values from flux-surface GENE simulations. Here $P_{i}$ is the ion pressure, $a$ is the minor radius of the torus, $r$ denotes the radial coordinate, $c_{i}$ is the ion sound speed and $\rho_{i}$ is the ion gyroradius.

the poloidal circumference, and the fluctuations cover almost the entire outboard side. In this case, the relevant parameter is $\rho_{c}^{*}=\rho_{i} / L_{c}$, which is much smaller than $\rho^{*}$, and therefore flux-surface tokamak simulations do not deviate significantly from the gyro-Bohm scaling.

These results have implications for the long-debated issue of transport stiffness. In tokamak experiments, the energy transport is usually observed to be stiff, in the sense that the heat flux is very small below a critical temperature gradient and increases sharply above it $[18,19]$. The temperature profile is therefore almost independent of the heating power and deposition profile but highly dependent on the boundary condition at the plasma edge [20]. In stellarators, a critical gradient is still observed, however no abrupt increase of the heat transport is usually reported [21-23]. This property, which has never received a generally accepted explanation, could be addressed by the GENE flux-surface simulations, suggesting a mild heat-flux scaling for sufficiently large $\rho^{*}$.

The possibility that reactor-sized stellarators, characterized by small $\rho^{*}$, might suffer from stiff transport makes it desirable to control turbulence by optimizing the magnetic geometry. To demonstrate the feasibility of this concept, we present in Fig. 3 a novel stellarator-field design, called MPX, as a modification to W7-X (specifically, its "high-mirror" configuration) in such a way that the ITG transport scaling is improved while excellent neoclassical confinement is maintained. The MPX design

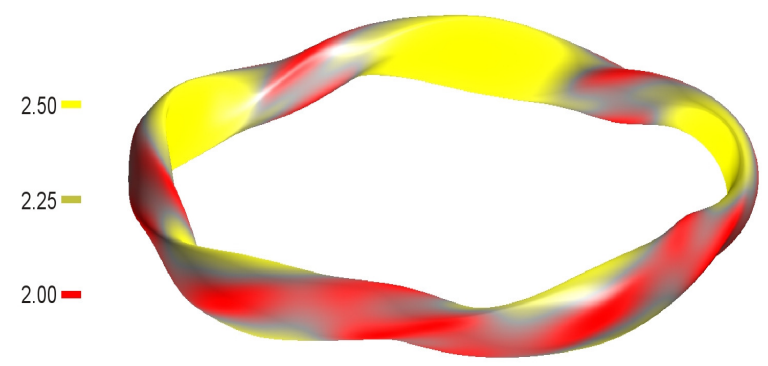

FIG. 3. The turbulence-optimized MPX stellarator design. Shown is the magnetic field strength (Tesla) over a magnetic surface. MPX is the first example of a quasi-omnigenous stellarator, similar to $\mathrm{W} 7-\mathrm{X}$, but with reduced turbulent transport. For this proof-of-principle configuration no attempt has been made to minimize the bootstrap current (which, however, takes acceptable values) or the alpha-particle losses.

was obtained by using the STELLOPT code to explore the configuration space by means of Differential Evolution, a population-based global search algorithm used previously for neoclassical optimization studies [24]. In order to reduce the ITG-mode intensity (the same principle holds also for electron-temperature-gradient-driven modes [25]), a cost function defined as $\chi_{I T G}^{2}=\kappa_{r}^{-}\left(g^{r r}\right)^{2}$, where $\kappa_{r}^{-}$is the negative part of the radial covariant component of the curvature and $g^{r r}$ the radial contraviariant metric element, was minimized. STELLOPT thus had the opportunity to suppress ITG transport by making 


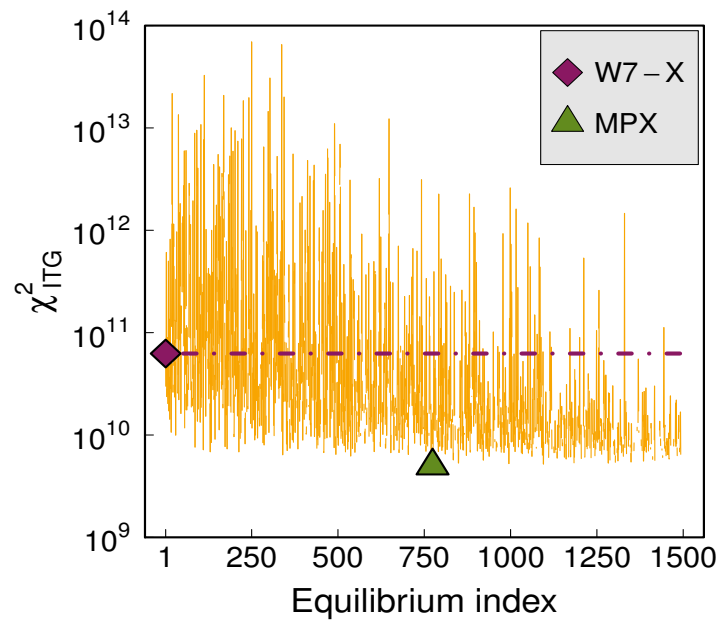

FIG. 4. (Color online) The MPX configuration was produced during a STELLOPT run equipped with the Differential Evolution algorithm. The fluctuations in the cost function represent the stochastic evolution of the population and diminish as the algorithm converges. MPX was singled out before the collisional transport (not shown here) started to increase.

the effective instability weaker, either by reducing the unfavorable (also known as "bad") curvature or by increasing the distance between flux surfaces in locations where the curvature is strong. The neoclassical transport was kept low by including the effective magnetic ripple $\epsilon_{\text {eff }}[26]$ in the total cost function.

In the STELLOPT optimization, shown in Fig. 4, it was found that about $3 / 4$ of the explored configurations had lower values of the cost function $\chi_{I T G}^{2}$ than W7-X, suggesting that for this device there is probably room for improvement with respect to ITG turbulence. Indeed, as can be seen in Fig. 5(a), MPX enjoys a weaker ITG transport scaling than W7-X. In addition, MPX performs better than W7-X also in its neoclassical optimization component thanks to its smaller magnetic ripple (see Fig. 5(b)). The overall dimensions of MPX and W7-X are almost identical but the changes to the magnetic field of W7-X imposed by STELLOPT are significant. The reduction in average elongation (defined as $\left(\epsilon_{t} / b_{1,0}\right)^{2}$, where $\epsilon_{t}$ is the ratio of the minor to major radius of the torus and $b_{1,0}$ the $(m=1, n=0)$ Fourier harmonic of the field strength) drops considerably. Although this change exacerbates the bad curvature, it also has the effect of pushing the flux surfaces apart, thus reducing the local temperature gradient in bad-curvature regions. Based on this insight, we selected two more configurations from the W7-X family, and carried out fluxsurface ITG simulations, in order to assess the effect of elongation on transport. As shown in Fig. 6, the configuration with the smaller elongation manifests a milder transport scaling, a feature which agrees with the STEL-

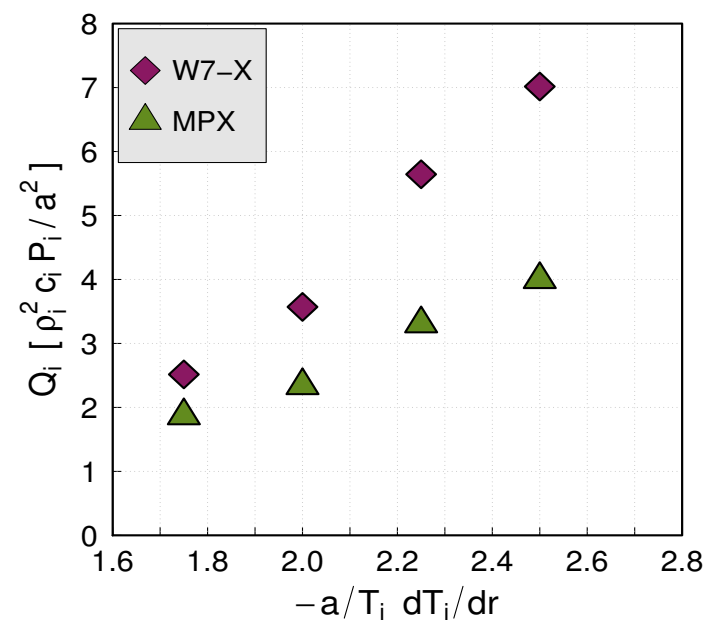

(a)

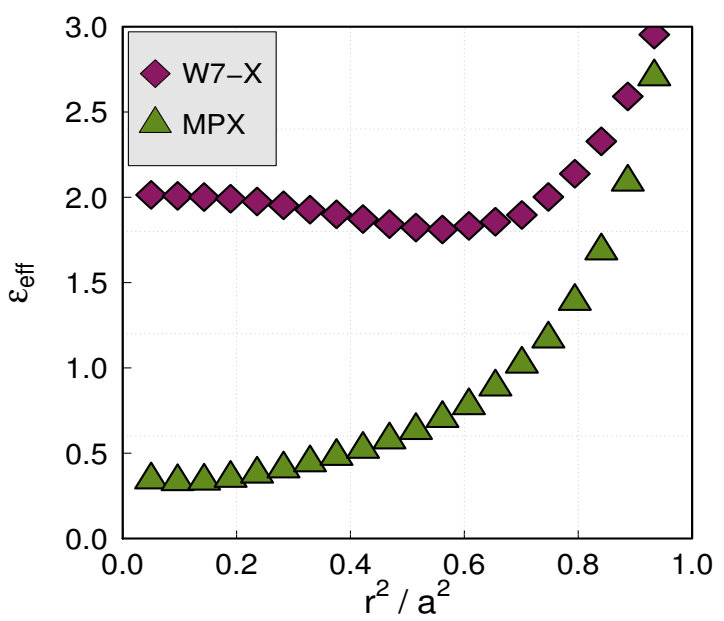

(b)

FIG. 5. (Color online) (a) Comparison of the ion-heat-flux scaling between MPX and W7-X with flux-surface GENE simulations. (b) Comparison of the neoclassical confinement between MPX and W7-X over the plasma radius.

LOPT prediction. At the same time, however, reducing the elongation led to the degradation of the neoclassical confinement. These results - which lend themselves to direct experimental testing - justify optimization efforts to design stellarators improved in both transport channels, as achieved in MPX.

The sensitivity of plasma turbulence to the magneticfield geometry is actually even broader than that suggested by the ITG analysis. If the electrons are also treated gyrokinetically, recent analytical theory [27-29] and numerical results [30] show that densitygradient-driven instabilities are much more stable in many stellarators than in tokamaks. The reason is that these modes are caused by electrons being magnetically 


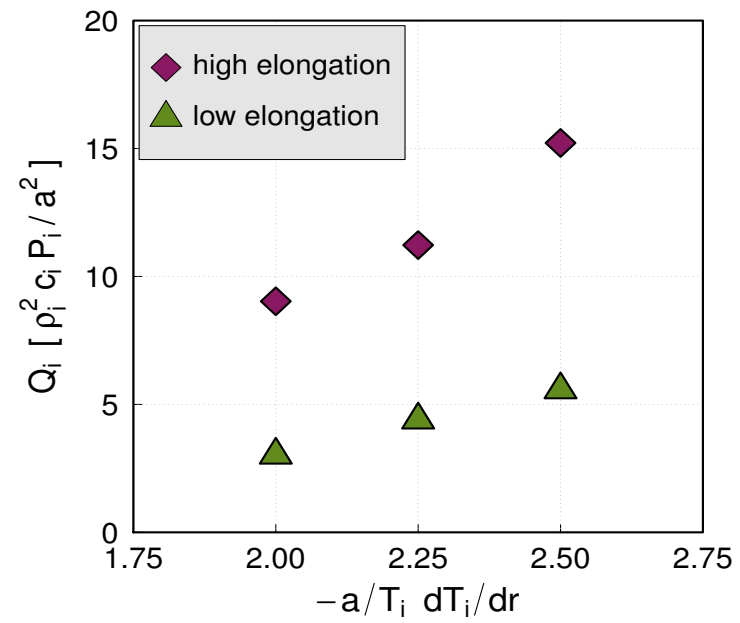

FIG. 6. (Color online) Comparison of the ion-heat-flux scaling between two W7-X configurations with different elongations. Similar to MPX, the less elongated configuration is associated with a weaker transport scaling.

trapped in regions of bad curvature. In tokamaks, the trapping regions are located exactly where the magneticfield curvature is bad (on the outboard side of the torus), while in some stellarators the trapping and bad-curvature regions can be separated from each other. In W7-X and MPX, these regions have small overlap, and linear gyrokinetic simulations show that MPX, too, is resilient against trapped-electron instabilities.

In conclusion, we showed that the distribution of turbulence fluctuations on a stellarator magnetic surface follows a rather localized pattern compared to a tokamak, thus suggesting a plausible explanation for the non-stiff transport observed in present stellarator experiments. This attractive feature is however expected to be minor in future reactor-sized devices, stimulating efforts toward further optimizing stellarator magnetic fields for reduced turbulence. In this spirit, we documented a new proof-of-principle configuration with this property, based on W7-X. While this investigation focused on quasiomnigenous stellarators, the involved numerical tools can readily accommodate other optimizations (e.g., quasiaxisymmetric, quasi-helical) as well as tokamak configurations with 3-d magnetic-field perturbations.

The GENE simulations were performed on the Helios super-computer (Japan).
[1] T. E. Evans et al., Nat. Phys. 2, 419-423 (2006).

[2] Y. Camenen et al., Nucl. Fusion 47, 510-516 (2007).

[3] H. Yamada et al., Plasma Phys. Control. Fus. 43, A55A71 (2001).

[4] A. H. Boozer, Rev. Mod. Phys. 76, 1071-1141 (2005).

[5] H. E. Mynick, N. Pomphrey and P. Xanthopoulos, Phys. Rev. Lett. 105, 095004 (2010).

[6] T. Klinger et al., Fusion Eng. Des. 88, 461-465 (2013).

[7] M. A. Beer, S. C. Cowley and G. W. Hammett, Phys. Plasmas 2, 2687-2700 (1995).

[8] T. -H. Watanabe, H. Sugama and M. Nunami, Nucl. Fusion 51, 123003 (2011).

[9] P. Xanthopoulos, F. Merz, T. Görler and F. Jenko, Phys. Rev. Lett. 99, 035002 (2007).

[10] T. -H. Watanabe, H. Sugama and S. Ferrando-Margalet, Phys. Rev. Lett. 100, 195002 (2008).

[11] P. Xanthopoulos, A. Mischchenko, P. Helander, H. Sugama and T. -H. Watanabe, Phys. Rev. Lett. 107, 245002 (2011).

[12] M. Nunami, T. -H. Watanabe, H. Sugama and K. Tanaka, Phys. Plasmas 19, 042504 (2012).

[13] F. Jenko, W. Dorland, M. Kotschenreuther and B. N. Rogers, Phys. Plasmas 7, 1904-1910 (2000).

[14] P. Xanthopoulos et al., Phys. Plasmas 16, 082303 (2009).

[15] T. Görler et al., J. Comp. Phys. 230, 7053-7071 (2011).

[16] E. A. Freeman and L. Chen, Phys. Fluids 25, 502-508 (1982).

[17] D. H. E. Dubin, J. A. Krommes, C. Oberman and W. W. Lee, Phys. Fluids 26, 3524-3535 (1983).

[18] U. Stroth, Plasma Phys. Control. Fus. 40, 9-74 (1998).

[19] P. Mantica et al., Phys. Rev. Lett. 102, 175002 (2009).

[20] T. C. Luce and C. C. Petty, Nucl. Fusion 34, 121-130 (1994).

[21] F. Wagner et al., Phys. Rev. Lett. 56, 2187-2190 (1986).

[22] J. Sanchez and V. Tribaldos, Plasma Phys. Control. Fus. 47, B349-B361 (2005).

[23] M. Hirsch et al., Plasma Phys. Control. Fus. 50, 053001 (2008)

[24] H. E. Mynick, N. Pomphrey and S. Ethier, Phys. Plasmas 9, 869-876 (2002).

[25] H. E. Mynick, N. Pomphrey and P. Xanthopoulos, Phys. Plasmas 18, 056101 (2011).

[26] C. D. Beidler and H. Maaßberg, Plasma Phys. Control. Fus. 43, 1131-1148 (2001).

[27] P. Helander et al., Plasma Phys. Control. Fus. 54, 124009 (2012).

[28] J. H. E. Proll, P. Helander, J. W. Connor and G. G. Plunk, Phys. Rev. Lett. 108, 245002 (2012).

[29] P. Helander, J. H. E. Proll and G. G. Plunk, Phys. Plasmas 20, 122505 (2013).

[30] J. H. E. Proll, P. Helander and P. Xanthopoulos, Phys. Plasmas 20, 122506 (2013). 